\title{
Cluster of Parasite Infections by the Spatial Scan Analysis in Korea
}

\author{
Kyoung-Eun Bae', Yoon Kyung Chang'², Tong-Soo Kim³ ${ }^{3}$ Sung-Jong Hong ${ }^{4} \mathbb{D}$, Hye-Jin Ahn², Ho-Woo Nam², \\ Dongjae $\mathrm{Kim}^{1, *}$ (1) \\ ${ }^{1}$ Department of Biomedicine Health Science, College of Medicine, The Catholic University of Korea, Seoul 06591, Korea; ${ }^{2}$ Department of \\ Parasitology, College of Medicine, The Catholic University of Korea, Seoul 06591, Korea; ${ }^{3}$ Department of Parasitology and Tropical Medicine and \\ Inha Research Institute for Medical Sciences, Inha University School of Medicine, Incheon 22333, Korea; ${ }^{4}$ Department of Medical Environmental \\ Biology, Chung-Ang University College of Medicine, Seoul 06974, Korea
}

\begin{abstract}
This study was performed to find out the clusters with high parasite infection risk to discuss the geographical pattern. Clusters were detected using SatScan software, which is a statistical spatial scan program using Kulldorff's scan statistic. Information on the parasitic infection cases in Korea 2011-2019 were collected from the Korea Centers for Disease Control and Prevention. Clusters of Ascaris lumbricoides infection were detected in Jeollabuk-do, and T. trichiura in Ulsan, Busan, and Gyeongsangnam-do. C. sinensis clusters were detected in Ulsan, Daegu, Busan, Gyeongsangnamdo, and Gyeongsangbuk-do. Clusters of intestinal trematodes were detected in Ulsan, Busan, and Gyeongsangnam-do. $P$. westermani cluster was found in Jeollabuk-do. E. vermicularis clusters were distributed in Gangwon-do, Jeju-do, Daegu, Daejeon, and Gwangju. This clustering information can be referred for surveillance and control on the parasitic infection outbreak in the infection-prone areas.
\end{abstract}

Key words: Ascaris lumbricoides, Trichuris trichiura, cluster, Korea

\section{INTRODUCTION}

Parasitic infection rate in Republic of Korea has decreased ever since the national public health project (the Parasite Disease Prevention Act) took effect in 1966. According to National Survey on Parasitic Infection, which has been conducted every 5-7 years, the overall helminth egg positive rate was $2.6 \%$ in the 8th survey, decreased from $2.7 \%$ of the 7th survey, yet the estimated number of infections nationwide was about 1.3 million $[1,2]$. According to the 8th survey, the parasite of the highest infection rate was Clonorchis sinensis with the rate of $1.9 \%$, which took $70 \%$ from the total infection. This was a significant implication for parasite prevalence, meaning that parasite epidemic has been changed from soil-borne such as Ascaris lumbricoides and Trichuris trichiura to fish-borne infections [2]. In addition, there are still a significant number of cases of parasitic infection reported every year and complications of

\footnotetext{
- Received 13 July 2020, revised 19 October 2020, accepted 2 November 2020.

*Corresponding author (djkim@catholic.ac.kr)

(C) 2020, Korean Society for Parasitology and Tropical Medicine

This is an Open Access article distributed under the terms of the Creative Commons Attribution Non-Commercial License (https://creativecommons.org/licenses/by-nc/4.0) which permits unrestricted non-commercial use, distribution, and reproduction in any medium, provided the original work is properly cited.
}

these infections can develop into serious diseases. Therefore, it is necessary to find a cluster where infection risk is significantly high and discuss the prevalence pattern to monitor and prevent the parasite infection in susceptible areas. To detect a cluster, Kulldorffs SaTScan Software was used, which provides computational resource that enables researchers to locate and statistically test the clusters [3].

\section{MATERIALS AND METHODS}

\section{Ethics statement}

This study was performed under the regulation of the IRB Committee of The Catholic University of Korea (No. MIRB20200529-002). This research adhered to the tenets of the Declaration of Helsinki.

\section{Data Source and definition}

The nationwide parasitic infection cases reported for 9 years (2011-2019) were collected from 16 administrative districts by the Korea Disease Control and Prevention Agency (KCDA) [4]. The parasitic infections were of Ascaris lumbricoides, Trichuris trichiura, Clonorchis sinensis, Paragonimus westermani, intestinal trematodes and Enterobius vermicularis. 
The population data of 16 administrative divisions 20112019 were obtained from the Korean Statistical Information Service [5]. The geographical locations of the infection cases were set to the latitude and longitude of administrative center as the search point since the case data were compiled by administrative district unit. The population and number of parasitic infection cases were summed up as accumulated counts and numbers for the 9 years by administrative district (Table 1).

\section{Spatial scan statistical analyses}

SaTScan Software using the spatial scan statistic [3] were employed to cluster the parasitic infection cases and to evaluate statistical significance of the located clusters through simulation. The discrete Poisson model was used since the parasitic infection case data were linked to their background population at risk [6]. The spatial scan statistic detected clusters by creating a circular window on a map and scanning the study area by varying a window size. The null hypothesis is that disease risk of both inside and outside of the window are the same, while the alternative hypothesis is that disease risk within the window is different from the outside [7]. A likelihood ratio for every window was calculated and the maximum likelihood ratio was defined as the test statistic. The window with the maximum likelihood ratio was identified as the most likely cluster, the significant cluster which is least likely to have occurred by chance while other windows were classified as sec- ondary clusters. The window size determines a percentage of the population at risk within the window and SaTscan Software provides its users an option to set maximum cluster size, the upper bound of window size. [6,8]. By increasing window size, its radius expands until the risk population within the window reaches the set value. If other administrative districts are located within the radius, the cluster is created by combining 2 districts into one and becomes larger cluster [7]. Therefore, increase of the window size resulted in larger clusters by merging of adjacent administrative districts in the radius [7,9]. It is important to find appropriate set value of cluster size because large value could hide effect of small core clusters, and small value could overlook the regional pattern of clusters [10]. This study adapted $5 \%, 15 \%$, and $25 \%$ as the values to spot the cluster in different window size. Statistical significance of the clusters was calculated using the Monte Carlo simulations [11,12] and expressed as p-value. A significance level of alpha $<0.05$ was used as a standard. R program (ver. 4.0.1) was used to visualize cluster patterns on a map. The clusters were numbered in order of likelihood ratio. This study analyzed accumulated data for 9 years, 2011-2019, without taking time into account.

\section{RESULTS}

\section{General clustering}

With 5\% maximum cluster size, Ulsan, Daegu, Jeju-do, and

Table 1. The cumulated parasite infection cases and population for 2011-2019 by administrative district

\begin{tabular}{|c|c|c|c|c|c|c|c|}
\hline \multirow[b]{2}{*}{ District (no.) } & \multirow[b]{2}{*}{ Population } & \multicolumn{6}{|c|}{ No. of infection cases of } \\
\hline & & $\begin{array}{c}\text { Ascaris } \\
\text { lumbricoides }\end{array}$ & $\begin{array}{l}\text { Trichuris } \\
\text { trichiura }\end{array}$ & $\begin{array}{l}\text { Clonorchis } \\
\text { sinensis }\end{array}$ & $\begin{array}{l}\text { Intestinal } \\
\text { trematodes }\end{array}$ & $\begin{array}{c}\text { Paragonimus } \\
\text { westermani }\end{array}$ & $\begin{array}{l}\text { Enterobius } \\
\text { vermicularis }\end{array}$ \\
\hline Seoul (1) & $89,996,828$ & 17 & 158 & 421 & 59 & 6 & 185 \\
\hline Busan (2) & $31,474,736$ & 3 & 514 & 5,265 & 898 & 0 & 11 \\
\hline Daegu (3) & $22,355,184$ & 1 & 114 & 1,849 & 178 & 1 & 226 \\
\hline Incheon (4) & $26,156,739$ & 6 & 41 & 87 & 5 & 0 & 10 \\
\hline Gwangju (5) & $13,202,461$ & 1 & 17 & 479 & 33 & 1 & 121 \\
\hline Daejeon (6) & $13,604,984$ & 9 & 92 & 227 & 27 & 0 & 134 \\
\hline Ulsan (7) & $10,420,219$ & 0 & 93 & 2,739 & 169 & 0 & 63 \\
\hline Gyeonggi-do (8) & $113,053,274$ & 4 & 152 & 279 & 60 & 2 & 192 \\
\hline Gangwon-do (9) & $13,896,792$ & 1 & 5 & 16 & 2 & 0 & 788 \\
\hline Chungcheongbuk-do (10) & $14,249,464$ & 0 & 6 & 217 & 28 & 0 & 10 \\
\hline Chungcheongnam-do (11) & $18,781,102$ & 0 & 0 & 0 & 0 & 0 & 8 \\
\hline Jeollabuk-do (12) & $16,736,755$ & 15 & 32 & 188 & 44 & 7 & 47 \\
\hline Jeollanam-do (13) & $17,097,958$ & 0 & 0 & 20 & 0 & 0 & 2 \\
\hline Gyeongsangbuk-do (14) & $24,235,379$ & 0 & 0 & 146 & 52 & 0 & 0 \\
\hline Gyeongsangnam-do (15) & $30,167,674$ & 4 & 538 & 4,728 & 1,988 & 1 & 24 \\
\hline Jeju-do (16) & $5,622,276$ & 0 & 0 & 3 & 1 & 0 & 551 \\
\hline Total & $461,051,825$ & 61 & 1,762 & 16,664 & 3,544 & 18 & 2,372 \\
\hline
\end{tabular}


Gangwon-do were clustered. Ulsan was the most likely cluster (LLR) with relative risk (RR) of 6.2, indicating that total parasite infection risk in Ulsan was 6.2 times higher than in the other districts. Followed were Daegu, Jeju-do and Gangwondo in order of their likelihood ratio. With 15\% maximum window size, Busan and Gyeongsangnam-do was the most likely cluster, indicating parasitic infection risk in Busan and Gyeongsangnam-do area was 8.7 times higher than other areas. With 25\% maximum window size, Busan, Gyeongsangnam-do, Ulsan and Daegu were the most likely cluster, indicating the total parasite infection risk in these districts was 15 times higher than in the other districts (Table 2). The general parasitic infection 2011-2019 showed that core outbreaks were prominent in Ulsan, Daegu, and Jeju-do while the regional outbreak pattern was found in the southeastern region such as Busan, Gyeongsangnam-do, Ulsan, and Daegu (Fig. 1).

\section{Clusters of Ascaris lumbricoides and Trichuris trichiura}

With 5\% maximum cluster size, the cases of A. lumbricoides were most likely cluster in Jeollabuk-do, indicating the risk of A. lumbricoides infection was 8.7 times higher in Jeollabuk-do than other areas. The secondary cluster was Daejeon. With $15 \%$ of maximum cluster size, the most likely cluster was Jeollabuk-do and Daejeon. Clustering with 25\% window was same to that of $15 \%$. The cases of A. lumbricoides infection were mainly distributed in Jeollabuk-do.

T. trichiura with 5\% maximum cluster size, the most likely cluster was Ulsan with relative risk of 2.4 while secondary clusters were Daejeon and Daegu. With 15\% maximum cluster size, Busan and Gyeongsangnam-do was the most likely cluster with relative risk of 9.6, indicating T. trichiura infection risk was 9.6 times higher in Busan and Gyeongsangnam-do. The secondary cluster was Daejeon. With 25\% maximum cluster size, Busan, Gyeongsangnam-do and Ulsan was the most like-

Table 2. Clusters detected from general parasite infection by window size

\begin{tabular}{|c|c|c|c|c|c|}
\hline Window Size & District & Case No. & Relative risk & Log likelihood ratio & $P$-value \\
\hline \multirow[t]{4}{*}{$5 \%$} & Ulsan & 3,064 & 6.2 & $2,876.845$ & $<0.001$ \\
\hline & Daegu & 2,369 & 2.11 & 488.693 & $<0.001$ \\
\hline & Jeju-do & 555 & 1.88 & 89.688 & $<0.001$ \\
\hline & Gangwon-do & 812 & 1.11 & 3.908 & 0.024 \\
\hline \multirow[t]{2}{*}{$15 \%$} & Busan, Gyeongsangnam-do & 13,974 & 8.67 & $12,945.557$ & $<0.001$ \\
\hline & Jeju-do & 555 & 1.88 & 89.688 & $<0.001$ \\
\hline $25 \%$ & Busan, Gyeongsangnam-do, Ulsan, Daegu & 19,407 & 15.03 & $19,526.059$ & $<0.001$ \\
\hline
\end{tabular}

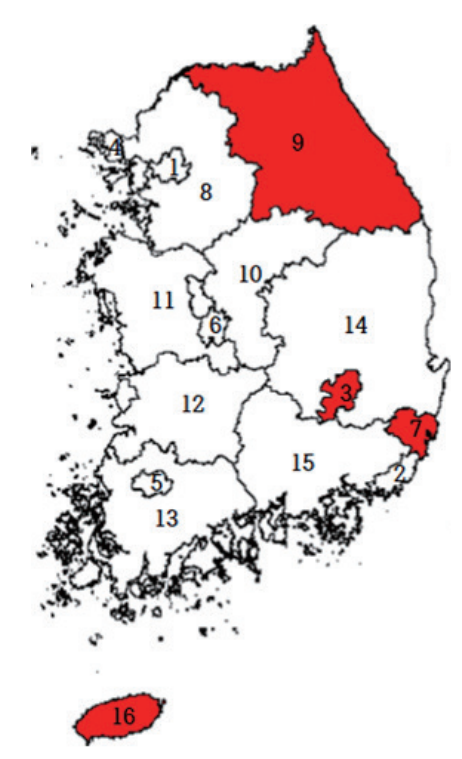

Window size $5 \%$

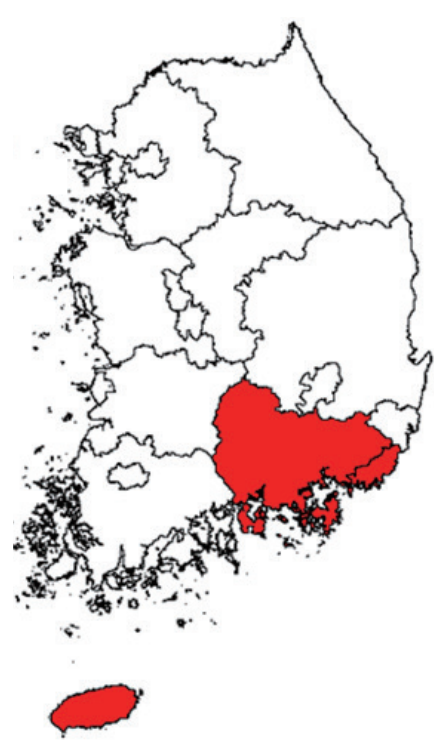

Window size $15 \%$

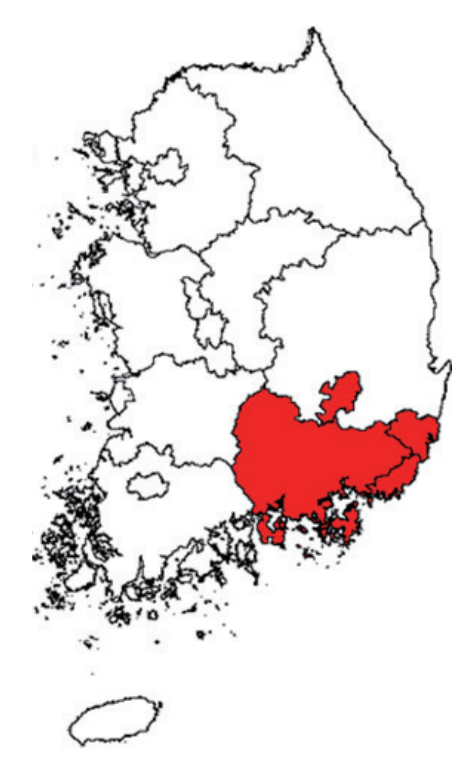

Window size $25 \%$

Fig. 1. Clusters detected in general parasitic infections by window size. Numbers identifying the administrative districts are referred to Table 1. 
Table 3. Clusters detected from Ascaris lumbricoides and Trichuris trichiura infections by window size

\begin{tabular}{|c|c|c|c|c|c|}
\hline Window size & District & Case No. & Relative risk & Log likelihood ratio & $P$-value \\
\hline \multicolumn{6}{|c|}{ Ascaris lumbricoides } \\
\hline \multirow[t]{2}{*}{$5 \%$} & Jeollabuk-do & 15 & 8.66 & 17.414 & $<0.001$ \\
\hline & Daejeon & 9 & 5.69 & 7.742 & $<0.001$ \\
\hline $15 \%$ & Jeollabuk-do, Daejeon & 24 & 9.21 & 26.936 & $<0.001$ \\
\hline $25 \%$ & Jeollabuk-do, Daejeon & 24 & 9.21 & 26.936 & $<0.001$ \\
\hline \multicolumn{6}{|c|}{ Trichuris trichiura } \\
\hline \multirow[t]{3}{*}{$5 \%$} & Ulsan & 93 & 2.41 & 26.531 & $<0.001$ \\
\hline & Daejeon & 92 & 1.81 & 12.966 & $<0.001$ \\
\hline & Daegu & 114 & 1.36 & 4.562 & 0.011 \\
\hline \multirow[t]{2}{*}{$15 \%$} & Busan, Gyeongsangnam-do & 1,052 & 9.6 & $1,030.771$ & $<0.001$ \\
\hline & Daejeon & 92 & 1.81 & 12.966 & $<0.001$ \\
\hline \multirow[t]{2}{*}{$25 \%$} & Busan, Gyeongsangnam-do, Ulsan & 1,145 & 10.02 & $1,088.969$ & $<0.001$ \\
\hline & Daejeon & 92 & 1.81 & 12.966 & $<0.001$ \\
\hline
\end{tabular}

Table 4. Clusters detected from Clonorchis sinensis, intestinal trematodes and Paragonimus westermani by window size

\begin{tabular}{|c|c|c|c|c|c|}
\hline Window size & District & Case No. & Relative risk & Log likelihood ratio & $P$-value \\
\hline \multicolumn{6}{|c|}{ Clonorchis sinensis } \\
\hline \multirow[t]{2}{*}{$5 \%$} & Ulsan & 2,739 & 8.51 & 3252.351 & $<0.001$ \\
\hline & Daegu & 1,849 & 2.45 & 524.628 & $<0.001$ \\
\hline \multirow[t]{2}{*}{$15 \%$} & Busan, Gyeongsangnam-do & 9,993 & 9.71 & 9847.711 & $<0.001$ \\
\hline & Gyeongsangbuk-do, Daegu & 1,995 & 1.21 & 30.359 & $<0.001$ \\
\hline $25 \%$ & Busan, Gyeongsangnam-do, Ulsan, Daegu & 14,581 & 27.18 & 17321.082 & $<0.001$ \\
\hline \multicolumn{6}{|c|}{ Intestinal trematodes } \\
\hline $5 \%$ & Ulsan & 169 & 2.17 & 38.432 & $<0.001$ \\
\hline $15 \%$ & Busan, Gyeongsangnam-do & 2,886 & 28.42 & 4200.849 & $<0.001$ \\
\hline $25 \%$ & Busan, Gyeongsangnam-do, Ulsan & 3,055 & 33.72 & 4330.98 & $<0.001$ \\
\hline \multicolumn{6}{|c|}{ Paragonimimus westermani } \\
\hline $5 \%$ & Jeollabuk-do & 7 & 16.89 & 11.5896 & $<0.001$ \\
\hline $15 \%$ & Jeollabuk-do & 7 & 16.89 & 11.5896 & $<0.001$ \\
\hline $25 \%$ & Jeollabuk-do & 7 & 16.89 & 11.5896 & $<0.001$ \\
\hline
\end{tabular}

ly cluster with relative risk of 10, indicating T. trichiura infection in Busan, Gyeongsangnam-do and Ulsan area was 10 times higher than other regions (Table 3). Overall, 15\% and 25\% analysis showed that big clusters including Busan and Gyeongsangnam-do region had notably high risk of indicating T. trichiura infection.

\section{Clusters of Clonorchis sinensis, intestinal trematodes and Paragonimus westermani}

C. sinensis with $5 \%$ maximum cluster size, Ulsan was the most likely cluster with relative risk of 8.5 , indicating C. sinensis infection in Ulsan was 8.5 times higher than other areas. The secondary cluster was Daegu. With 15\% maximum cluster size, Busan and Gyeongsangnam-do was the most likely cluster with relative risk of 9.7. Gyeongsangbuk-do and Daegu was the secondary cluster. With 25\% maximum cluster size, Busan,
Gyeongsangnam-do, Ulsan and Daegu were the most likely cluster with relative risk of 27.2. Spatial scan analysis on the $C$. sinensis-infected cases showed a consistent pattern in every cluster size that the $C$. sinensis infection risk was prominent in Gyeongsangnam-do (Table 4).

For intestinal trematodes, with 5\% maximum cluster size, the most likely cluster was Ulsan with relative risk of 2.2, indicating that the intestinal trematode infections in Ulsan was 2.2 times higher than in the other areas. With 15\% maximum cluster size, the most likely cluster was Busan and Gyeongsangnam-do with relative risk of 28.4. With 25\% maximum cluster size, the most likely cluster was Busan, Gyeongsangnam-do and Ulsan with relative risk of 33.7. In summary, the intestinal trematode infection was of high risk in Gyeongsangnam-do region.

The clusters of P. westermani infections with 5\%,15\%, and 
Table 5. Clusters detected from Enterobius vermicularis infection by window size

\begin{tabular}{|c|c|c|c|c|c|}
\hline Window size & District & Case No. & Relative risk & Log likelihood ratio & $P$-value \\
\hline \multirow[t]{5}{*}{$5 \%$} & Gangwon-do & 788 & 16.01 & 1,299.982 & $<0.001$ \\
\hline & Jeju-do & 551 & 24.51 & $1,164.773$ & $<0.001$ \\
\hline & Daegu & 226 & 2.07 & 44.449 & $<0.001$ \\
\hline & Daejeon & 134 & 1.97 & 23.916 & $<0.001$ \\
\hline & Gwangju & 121 & 1.82 & 17.406 & $<0.001$ \\
\hline \multirow[t]{5}{*}{$15 \%$} & Gangwon-do & 788 & 16.01 & $1,299.982$ & $<0.001$ \\
\hline & Jeju-do & 551 & 24.51 & $1,164.773$ & $<0.001$ \\
\hline & Daegu & 226 & 2.07 & 44.449 & $<0.001$ \\
\hline & Daejeon & 134 & 1.97 & 23.916 & $<0.001$ \\
\hline & Gwangju & 121 & 1.82 & 17.406 & $<0.001$ \\
\hline \multirow[t]{5}{*}{$25 \%$} & Gangwon-do & 788 & 16.01 & $1,299.982$ & $<0.001$ \\
\hline & Jeju-do & 551 & 24.51 & $1,164.773$ & $<0.001$ \\
\hline & Daegu & 226 & 2.07 & 44.449 & $<0.001$ \\
\hline & Daejeon & 134 & 1.97 & 23.916 & $<0.001$ \\
\hline & Gwangju & 121 & 1.82 & 17.406 & $<0.001$ \\
\hline
\end{tabular}

$25 \%$ window size were all same. The most likely cluster was in Jeollabuk-do with relative risk of 16.9.

\section{Clusters of Enterobius vermicularis}

With 5\% maximum cluster size, Gangwon-do was the most likely cluster with relative risk of 16 , indicating E. vermicularis infection risk in Gangwon-do was 16 times higher than in the other regions. The secondary cluster was in Jeju-do followed by Daegu, Daejeon and Gwangju. Jeju-do, however, showed exceptionally relative risk of 24.51 (Table 5). In E. vermicularis infection analysis, the results of $15 \%$ and $25 \%$ windows were the same to that of $5 \%$ window. The E. vermicularis infections distributed all over the study regions, while Gangwon-do and Jeju-do showed high risk of enterobiasis.

\section{DISCUSSION}

This study has provided the clusters with high risk of parasitic infection in Korea from 2011 to 2019, by using spatial scan analysis. Depending on the transmission of infections, the infections are respectively divided into soil-borne, fishborne, and contact-borne.

A. lumbricoides and T. trichiura are soil-transmitted helminths by ingesting worm eggs in the soil. According to Nationwide Survey on the Prevalence of Intestinal Parasitic Infections, A. lumbricoides showed a dramatic decrease of egg positive rate from $54.9 \%$ in the 1 st survey in 1971 to $0.03 \%$ in the 8 th survey in 2012 and T. trichiura from $65.4 \%$ to $0.4 \%$ [13]. The tendency of decrease also showed in this study that the soil-trans- mitted helminths infection cases comprised $7.5 \%$ of the data and especially with 61 reported A. lumbricoides cases nationwide in 9 years (Table 1). A. lumbricoides clusters were located in the southern-west and T. trichiura clusters, mostly in southern-east. The most T. trichiura infection-prone area consisted of Busan, Gyeongsangnam-do, Ulsan. Daejeon was also infection-prone area for A. lumbricoides and T. trichiura by being an overlapping cluster of both infections. The successful control over soil-transmitted helminths was carried out as economic growth of Korean society brought improvement of sanitary condition. Also, the use of chemical fertilizers on farm brought the change since traditional agricultural industry in 1970s used human manure as a fertilizer causing the widespread worm eggs in the soil and agricultural products growing on it [13].

C. sinensis, intestinal trematodes, and P. westermani are the fish-borne parasites transmitted by ingesting undercooked fresh-water fish and shellfish. The geographical locations of $C$. sinensis and intestinal trematodes infection-prone area was southern-east region including Busan, Gyeongsangnam-do, Ulsan while P. westermani was located in Jeollabuk-do (Fig. 2). The 3 fish-borne parasites consisted about $83 \%$ of the reported case data showing the prevalence of consuming raw or undercooked food. This culinary tradition came from the previous generation who had fresh-water fish and shellfish as a source of protein during famine. In addition, the tradition remains and is enjoyed by people in all generation. Therefore, reported cases could be infections from both past and present. The 7th survey in 2004 showed that fish-borne parasitic infection was steadily prevalent along the riverside areas [13]. 


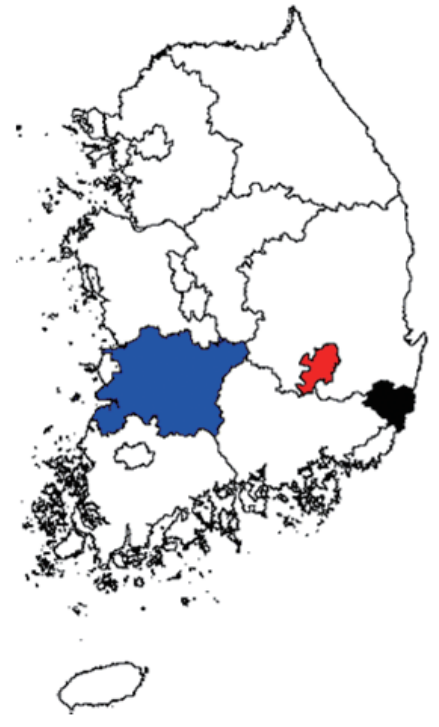

Window size $5 \%$

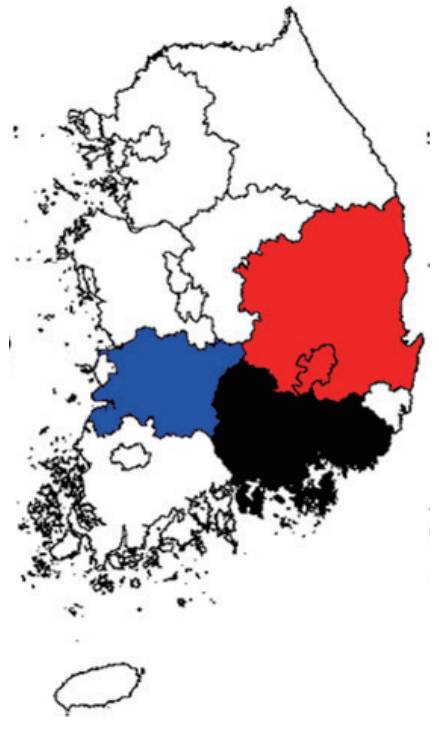

Window size $15 \%$

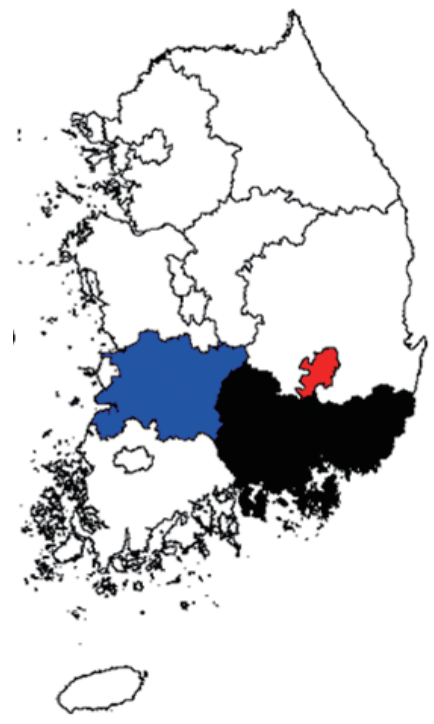

Window size $25 \%$

Fig. 2. Clusters detected from Clonorchis sinensis (red), intestinal trematodes, and Paragonimus westermani (blue) infections by window size. Clusters overlapped with $C$. sinensis and intestinal trematode infections are colored black.

E. vermicularis, also known as the pinworm, is transmitted by physical contact. The geographical clusters of Enterobius vermicularis were distributed all over the region. It is the common infection among preschool or school-age children and since the government is expanding the pre-school education, it is more likely to be increased [1].

\section{CONFLICT OF INTEREST}

The authors declare no conflict of interest related to this study.

\section{REFERENCES}

1. Korea Centers for Disease Control and Prevention, Korea Association of Health Promotion. Prevalence of Intestinal Parasitic Infection in Korea. The 7th Report. Seoul, Korea. 2004.

2. Korea Centers for Disease Control and Prevention. Korea Association of Health Promotion: Prevalence of intestinal parasitic infection in Korea -The 8th Report. Seoul, Korea. 2012.

3. Kulldorff M. A spatial scan statistic. Communications in Statistics-Theory and Methods. 1997; 26: 1481-1496.

4. Infectious Disease Portal. Parasitic infection statistics [Internet]; [cited 2020 Feb 13]. Available from: https://www.cdc.go.kr/npt/ biz/npp/iss/parasitosisStatisticsMain.do

5. Korean Statistical Information Service. Resident Population by
City, County, and District [Internet]; [cited 2020 Mar 12]. Available from: http://kosis.kr/statHtml/statHtml.do?orgId=101\&tblI d=DT_1B040A3\&conn_path=I3

6. Kulldorff M. SaTScan TM. User Guide for version 9.6. 2018.

7. Lee KJ, Hwang MH, Han SH, Yang EJ. A First steps for understanding and using spatial statistics analysis. Korea Research Institute for Human Settlements 2015.

8. Kulldorff M, Nagarwalla N. Spatial disease clusters: detection and inference. Stat Med 1995; 14: 799-810. https://doi.org/10.1002/ sim.4780140809

9. Han JH, Lee MJ. Cancer cluster detection using scan statistic. J Korean Data Inf Sci Soc 2016; 27: 1193-1201 (in Korean). https:// doi.org/10.7465/jkdi.2016.27.5.1193

10. Chen J, Roth RE, Naito AT, Lengerich EJ, Maceachren AM. Geovisual analytics to enhance spatial scan statistic interpretation: an analysis of U.S. cervical cancer mortality. Int J Health Geogr 2008; 7: 57. https://doi.org/10.1186/1476-072X-7-57

11. Kulldorff M. An isotonic spatial scan statistic for geographical disease surveillance. J Nat Inst Publ Health 1999; 48: 94-101.

12. Azage M, Kumie A, Worku A, Bagtzoglou AC. Childhood diarrhea exhibits spatiotemporal variation in northwest Ethiopia: a SaTScan spatial statistical analysis. PLoS One 2015; 10: e0144690. https://doi.org/10.1371/journal.pone.0144690

13. Kim TS, Cho SH, Huh S, Kong Y, Sohn WM, Hwang SS, Chai JY, Lee SH, Park YK, Oh DK, Lee JK. A nationwide survey on the prevalence of intestinal parasitic infections in the Republic of Korea 2004. Korean J Parasitol 2009; 47: 37-47. https://doi.org/10.3347/ kjp.2009.47.1.37 